\title{
A small difference in nuclear mass could make a big impact in the cosmos
}

\author{
SUN Yang \\ Department of Physics, Shanghai Jiao Tong University, Shanghai 200240, China
}

In a recent paper [1] published in Physical Review Letters, scientists at the Institute of Modern Physics, Chinese Academy of Sciences, have reported their first results from the Cooler Storage Ring at the Heavy Ion Research Facility, a newly-constructed major scientific facility in Lanzhou, China. As a part of an international collaboration including colleagues from Europe, USA and Japan, they were able to identify in experiments the very exotic nuclei ${ }^{63} \mathrm{Ge},{ }^{65} \mathrm{As}$, ${ }^{67} \mathrm{Se}$ and ${ }^{71} \mathrm{Kr}$ from about 700000 stored ions, and successfully measured their masses for the first time. Previously, data from test experiments had uncertainty errors that were too large [2] for any definite conclusions to be drawn [3]. In contrast to the majority of medium-mass and heavy nuclei in the universe, the nuclei reported in [1] are exotic, in the sense that the proton number $Z$ is greater than the neutron number $N$ in each nucleus; more specifically, $N=Z-1$. This is a very rare situation where the nuclear force can still hold individual nucleons to form an atomic nucleus despite the strong Coulomb repulsion. Nevertheless, such proton-rich nuclei are unstable and very short-lived. The half-life of ${ }^{65} \mathrm{As}$, for example, is only $128 \mathrm{~ms}$, meaning that in less than one second after they are created, half of the ${ }^{65}$ As isotopes disappear.

Weighing masses for very short-lived nuclei is extremely difficult. Even for the most advanced laboratories in the world today, such measurements are out of reach. For many years, only theoretical masses for these nuclei were listed in the nuclear mass table [4]. No one knew their experimental values. But why do we need to care about the exact masses of these nuclei that only exist, on average, for less than a second?

In practice, such short-lived nuclei are indeed not very useful. However, knowing their exact masses is crucial in understanding the processes involved in energy release and element production in the universe. The origin of heavy

email: sunyang@sjtu.edu.cn elements (heavier than iron) has been one of the major unsolved puzzles in physics [5]. The point that $\mathrm{Tu}$ et al. [1] emphasize is that a small correction in masses of the exotic nuclei can make a great impact on the question of the origin of elements in the cosmos. So where in the universe can such an impact happen, and why are these nuclear masses so important?

A century after Roentgen won the first Nobel Prize in 1901 for his discovery of X-rays, the prize was awarded to Riccardo Giacconi in 2002 for another X-ray-related work. The cosmic X-rays that Giacconi and his colleagues discovered were observed with space-based telescopes in the emissions of X-ray bursters. These are a class of X-ray binary stars exhibiting periodic and rapid increases in luminosity peaked in the X-ray region of the electromagnetic spectrum. The associated astrophysical systems are composed of an accreting neutron star and a companion, with material (rich in hydrogen and helium) from the companion star being transferred onto the neutron star surface. It is compressed and heated to extremely high temperatures so that thermonuclear runaway processes take place. The explosive stellar nucleosynthesis begins with the hot $\mathrm{CNO}$ cycle, which quickly leads to what is called the rapid proton capture process (or rp-process) [6]. In that process, protons fuse with seed nuclei, synthesizing isotopes near the proton drip-line - the boundary for nuclear existence beyond which nuclei simply cannot bind a further proton. The so-produced short-lived nuclei in the path eventually decay and populate nuclei towards the region of stability.

Simulations of nucleosynthesis in the rp-process require reliable nuclear physics input [6]. Nuclear mass, or more specifically the proton separation energy (the energy it takes to remove one proton from a nucleus), is the most important factor. If there are no measurements, theoretical mass values have to be used. Thus discussions over the rp-process nucleosynthesis rely heavily on the quality of these mass predictions. However, as pointed out by Tu et al. [1], such pre- 
dictions are not always reliable.

The key issue is precise mass values that link to the so-called waiting points [6]. A waiting point is a nucleus near the proton drip line where a captured proton is unbound or only weakly bound, and can easily be removed by photodisintegration. As a result, proton capture is effectively suppressed and the rp-process has to proceed via the slow $\beta^{+}$-decay. This delays nuclear burning and leads to the extended X-ray burst tail. Three major waiting points that dominate the energy generation rate during the burst tail have been discussed [6], namely ${ }^{64} \mathrm{Ge},{ }^{68} \mathrm{Se}$ and ${ }^{72} \mathrm{Kr}$. Their estimated long lifetimes provide the explanation for the observed burst tails. Detailed X-ray burst model calculations using the new mass value of ${ }^{65} \mathrm{As}[1]$ suggest that ${ }^{64} \mathrm{Ge}$ is no longer a good rp-process waiting point. This means that the better-defined nuclear physics parts of the process have now changed the traditional view of nucleosynthesis, leaving more possibilities to the later stages of element production in the X-ray burst environment. This would open up new research opportunities in astrophysics, nuclear physics and astronomical observations.

1 Tu X L, Xu H S, Wang M, et al. Direct mass measurements of the short-lived $A=2 Z-1$ nuclides ${ }^{63} \mathrm{Ge},{ }^{65} \mathrm{As},{ }^{67} \mathrm{Se}$ and ${ }^{71} \mathrm{Kr}$ and their impact on nucleosynthesis in the rp process. Phys Rev Lett, 2011, 106: 112501

$2 \mathrm{Xu} \mathrm{H} \mathrm{S,} \mathrm{Tu} \mathrm{X} \mathrm{L,} \mathrm{Yuan} \mathrm{Y} \mathrm{J,} \mathrm{et} \mathrm{al.} \mathrm{First} \mathrm{mass} \mathrm{measurement} \mathrm{of}$ short-lived nuclides at HIRFL-CSR. Chinese Sci Bull, 2009, 54: 4749-4752

3 Sun Y. Nuclear masses near the proton drip-line and their impact on nucleosynthesis in explosive stars. Chinese Sci Bull, 2009, 54: 4594-4595

4 Audi G, Wapstra A H, Thibault C. The AME 2003 atomic mass evaluation: (II). Tables, graphs and references. Nucl Phys A, 2003, 729: 337-676

5 Haseltine E. The 11 greatest unanswered questions of physics. Discovery, February 1, 2002

6 Schatz H, Aprahamian A, Goerres J, et al. rp-process nucleosynthesis at extreme temperature and density conditions. Phys Rep, 1998, 294: 167-263

Open Access This article is distributed under the terms of the Creative Commons Attribution License which permits any use, distribution, and reproduction in any medium, provided the original author(s) and source are credited. 